\title{
Effects of Polymer Network Surfaces on Expansion of Cholesteric Blue Phases Temperature*
}

\author{
Takeshi Noma, ${ }^{\dagger}$ Masayoshi Ojima, Hiroaki Asagi, Yuichi Kawahira, Akihiko Fujii, and Masanori Ozaki \\ Department of Electrical, Electronic and Information Engineering, \\ Graduate School of Engineering, Osaka University, \\ 2-1 Yamada-Oka, Suita, Osaka 565-0871, Japan \\ Hirotsugu Kikuchi \\ Institute for Materials Chemistry and Engineering, Kyushu University, \\ 6-1 Kasuga-Koen, Kasuga, Fukuoka 816-8580, Japan \\ (Received 12 October 2007; Accepted 27 December 2007; Published 25 January 2008)
}

\begin{abstract}
We proposed the stabilization of cholesteric blue phases (BPs) by confining liquid crystal (LC) molecules within a small area. The temperature range of the BP was expanded in the LC confined in the polymer network (PN). In particular, the temperature range of $\mathrm{BP} \mathrm{I}$ in the LC/PN composite is six times wider than that of the pure BP LC compound. Polarizing microscopic observations and field-dependence measurements of the expanded BP have been carried out, which indicate that it is in the metastable state and is induced by the pinning effect on the surfaces of the PN. [DOI: 10.1380/ejssnt.2008.17]
\end{abstract}

Keywords: Liquid Crystal; Cholesteric Blue Phase; Polymer Dispersed Liquid Crystal; Polymer Network; Pinning Effect

\section{INTRODUCTION}

The blue phases (BPs) of cholesteric liquid crystals have three-dimensional (3D) herical structures in a temperature range between a chiral nematic phase and an isotropic liquid phase [1], and exhibit many unique characteristics. For instance, they have a $3 \mathrm{D}$ periodic structure with a lattice constant in the order of visible wavelength [2] and include regularly ordered desclinations resulting from the 3D helicoidal molecular alignment. From the viewpoint of display application, since BPs are optically isotropic, display devices based on them should have a wide view angle and should not require an alignment process. In addition, because of their 3D helical structure with a periodicity in the order of visible wavelength, applications to photonic crystals [3-5] and fast electro-optical modulation [6-8] are also expected.

Despite their attractive characteristics, since BPs appear only in a narrow temperature range (less than a few degrees), their practical applications had been considered to be difficult. Recently, several attempts have been proposed to widen the temperature range of the BPs, which describe the stabilizations of the 3D cubic lattice by polymerizing disclination lines [9], and by using a flexoelectricity of twin molecules [10] and a chiral compound possessing molecular biaxiality [11].

In this study, we propose the stabilization of the BPs by using a pinning effect on surfaces of the polymer network $(\mathrm{PN})$. By forming a $\mathrm{PN}$ in the liquid crystal (LC), the temperature range of the $\mathrm{BPs}$ in the $\mathrm{LC} / \mathrm{PN}$ composite can be successfully extended.

\footnotetext{
*This paper was presented at Handai Nanoscience and Nanotechnology International Symposium (Handai Nano 2007), Osaka University, Japan, 26-28 September, 2007.

†Corresponding author: tnoma@opal.eei.eng.osaka-u.ac.jp
}

\section{EXPERIMENTAL}

The LC materials used in this study comprised a nematic mixture (JC-1041XX, Chisso Co.) and 4-cyano4'-pentylbiphenyl (5CB). A chiral dopant (ISO- $\left.(6 \mathrm{OBA})_{2}\right)$ was used to induce the BPs and a cholesteric phase. To form a PN in the LC, NOA65 (Norland Products) was used as a UV-curable monomer. Homogeneous mixtures of LC and monomer were prepared. The constituent fractions of the samples are listed in Table I. A glass cell was filled with a homogeneous mixture and irradiated with UV light (365 nm) passing through a bandpath UV filter for 10 min to induce a phase separation between the LCs and the polymer. The polymerization was performed in the isotropic phase at room temperature (approximately $20^{\circ} \mathrm{C}$ ).

\section{RESULTS AND DISCUSSION}

The optical textures of the samples in a sandwiched glass cell were observed by a polarizing optical microscope (Nikon) equipped with a hot stage (METTLER, FP90). The cell gap was maintained at $9 \mu \mathrm{m}$ by film spacers. For measuring the electric field-temperature phase diagram of the BP LC, glass substrates with indium-tin-oxide (ITO) transparent electrodes were used to apply an electric field perpendicular to the substrate surface, and a rectanglewave ac voltage $(1 \mathrm{kHz})$ was applied.

Figure 1 shows the scanning electron microscope (SEM)

TABLE I: Composition of samples used in preparing polymer network.

\begin{tabular}{|c|c|c|c|c|}
\hline \multirow{3}{*}{$\begin{array}{c}\text { Substance } \\
\text { Weight ratio/ } \\
\text { wt\% }\end{array}$} & \multicolumn{2}{|c|}{ Liquid crystal } & Chiral dopant & Monomer \\
\hline & $5 \mathrm{CB}$ & 1041XX & $\mathrm{ISO}-(6 \mathrm{OBA})_{2}$ & NOA65 \\
\hline & 46.3 & 46.3 & 75 & \\
\hline $\begin{array}{c}\text { Fraction in } \\
\text { composite/ wt } \%\end{array}$ & & 68.3 & & 31.7 \\
\hline
\end{tabular}




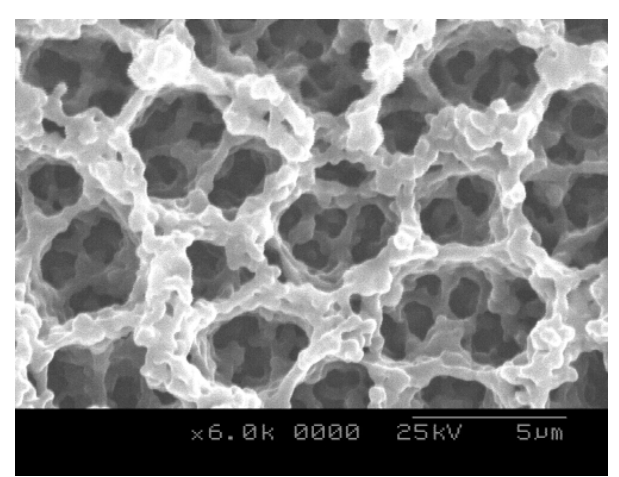

FIG. 1: SEM image of polymer network.

image of the LC/PN composite in which LCs were washed away. The $3 \mathrm{D} \mathrm{PN}$ is formed. The morphology of the polymer in the LC/PN composite depends on the constitution fraction of the LC and monomer. In general, when the ratio of the monomer to the LC is high, a polymer-dispersed liquid crystal (PDLC) structure should be formed. The LC-rich fraction in this study results in the formation of the network structure instead of the PDLC structure. The averaged size of the vacant spaces in the $\mathrm{PN}$ is approximately $5 \mu \mathrm{m}$, which can be controlled by changing the intensity of UV light or composition ratio of the LC and NOA65.

Figure 2 shows the polarizing microphotographs of the $\mathrm{BP}$ LC in the LC/PN composite. The sample was cooled from the isotropic phase at a cooling rate of $0.1^{\circ} \mathrm{C} / \mathrm{min}$. When the pure BP LC without the $\mathrm{PN}$ is cooled from the isotropic phase, blue phases-BP II and BP I-are sequentially observed in a narrow temperature range (42.3$45.2^{\circ} \mathrm{C}$ ) between the isotropic and cholesteric phases. As shown in Fig. 2, the BPs also appear in the LC/PN composite, although the platelet textures that are characteristic of the BP are small. However, it should be noted that the temperature range of the $\mathrm{BP}$ is drastically expanded. Even at $32^{\circ} \mathrm{C}$, the texture characteristic to BP I can be observed.

The phase transition temperatures of the BP LC without and with the PN are summarized in Fig. 3(a), which were measured when the sample was cooled from the isotropic phase at a cooling rate of $0.1^{\circ} \mathrm{C} / \mathrm{min}$. In the pure BP LC without the PN, BPs II and I were observed in a temperature range of $44.0-45.2^{\circ} \mathrm{C}$ and $42.3-44.0^{\circ} \mathrm{C}$, respectively. On the other hand, the $\mathrm{LC} / \mathrm{PN}$ composite shows BPs II and I in a wider temperature range of $39.2-41.0^{\circ} \mathrm{C}$ and $28.9-39.2^{\circ} \mathrm{C}$, respectively. The $\mathrm{BP}$ I temperature range of the $\mathrm{LC} / \mathrm{PN}$ composite is six times wider than that of the pure BP LC. We have also investigated the phase transition at a heating stage. Figure 3(b) shows the phase transition temperature of the pure BP $\mathrm{LC}$ and $\mathrm{LC} / \mathrm{PN}$ composite when the sample was heated from the cholesteric phase at a rate of $0.1^{\circ} \mathrm{C} / \mathrm{min}$. $\mathrm{Al}-$ though a slight expansion of the BP I temperature range is observed also in the heating stage, the large expansion observed during the cooling stage, as shown in Fig. 3(a), does not occur. Namely, the large expansion of the BPs in the network structure is monotropically observed only at the cooling stage, and BP I in the wide temperature range might be in a metastable state.

The influence of an electric field $E$ on the phase-

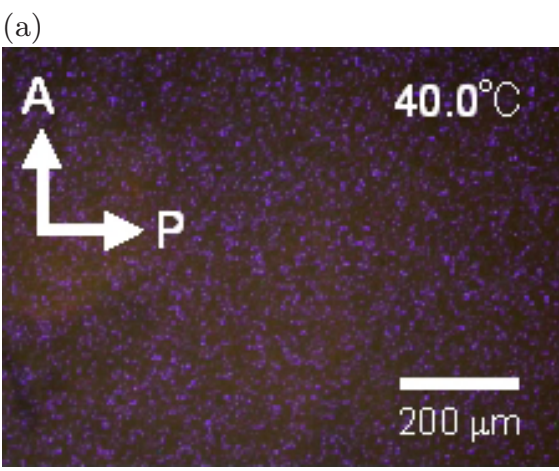

(b)

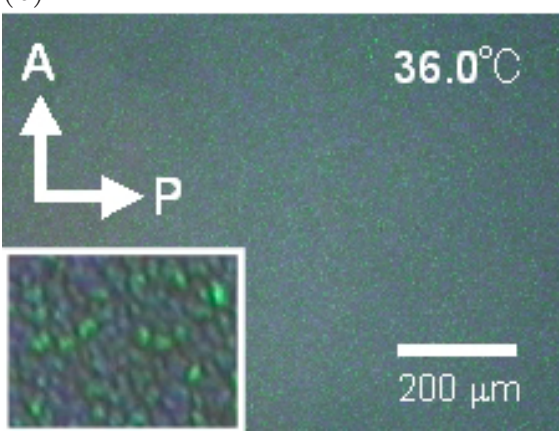

(c)

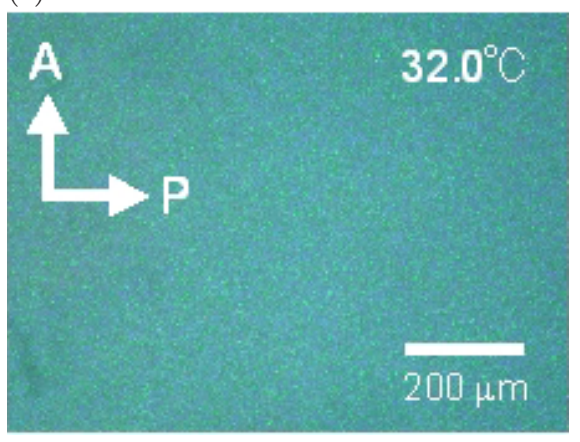

FIG. 2: Polarizing microscopic texture of (a) BP II at $40.0^{\circ} \mathrm{C}$; (b) BP I at $36.0^{\circ} \mathrm{C}$ and (c) $32.0^{\circ} \mathrm{C}$ on cooling from the isotropic liquid phase at a rate of $0.1^{\circ} \mathrm{C} / \mathrm{min}$.

transition temperature $T$ has been investigated. The $E-$ $T$ phase diagrams of the pure BP LC and LC/PN composite are shown in Fig. 4. The phase was determined by a polarizing microscope observation while the compound was cooled under a constant voltage at a cooling rate of $0.5^{\circ} \mathrm{C} / \mathrm{min}$. The temperature range of the $\mathrm{BP}$ in the $\mathrm{LC} / \mathrm{PN}$ composite (a) is significantly wider than that of the pure BP LC (b), which is consistent with the result shown in Fig. 3(a). Upon increasing the voltage, the BP I-cholesteric transition shifts toward a higher temperature, while the Iso-BP II and BP II-BP I transitions are almost independent of the applied voltage. These field dependences of the phase transition coincide with the results reported so far [12].

In order to confirm the metastability of the expanded $\mathrm{BP} \mathrm{I}$ in the $\mathrm{LC} / \mathrm{PN}$ composite, we have investigated a reversibility against the field application. As shown in Fig. 4, upon applying an electric field above the threshould, the BPs change to the cholesteric phase. At $38.9^{\circ} \mathrm{C}$, which is just below the $\mathrm{BP}$ I/BP II transition (shown as arrow A in Fig. 4(a)), BP I appears again after 


\section{(a) cooling}

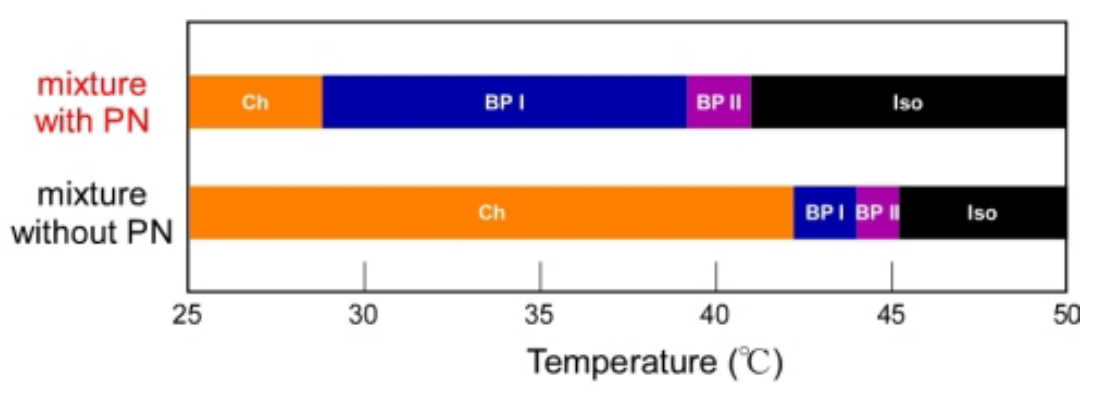

(b) heating

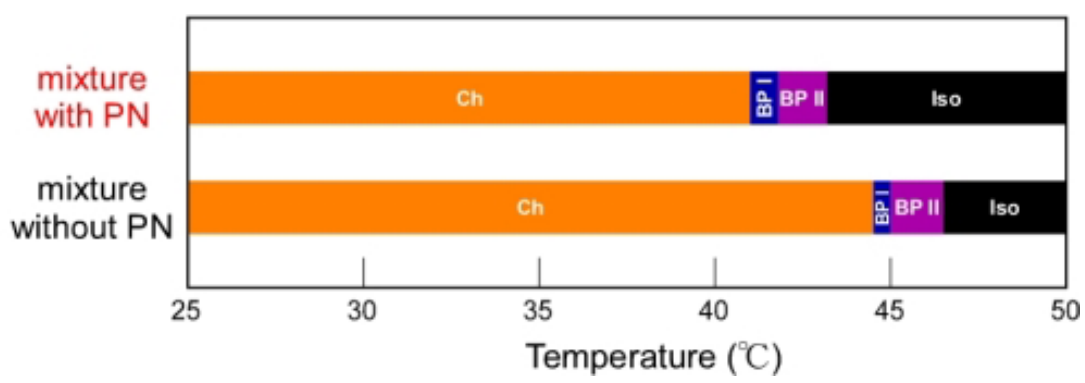

FIG. 3: Phase transition temperature of the BP liquid crystals with and without polymer network (a) on cooling (b) on heating at a rate of $0.1^{\circ} \mathrm{C} / \mathrm{min}$.

(a)

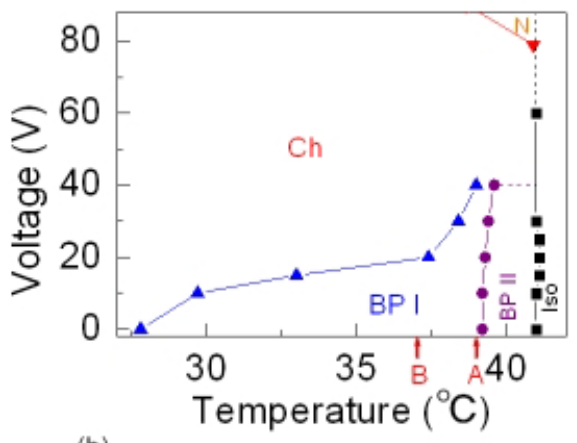

(b)

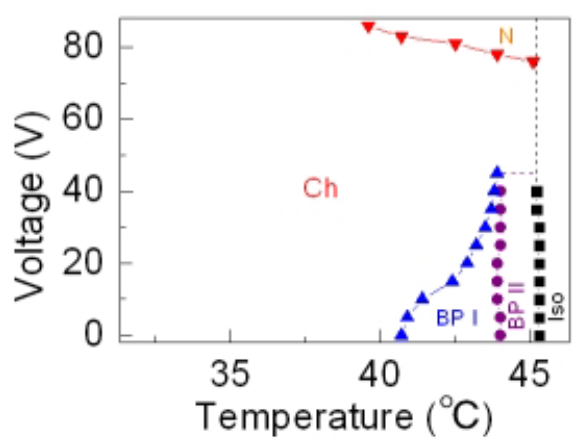

FIG. 4: Electric field-temperature phase diagrams of (a) $\mathrm{LC} / \mathrm{PN}$ composite and (b) pure BP liquid crystals.

removing the field. However, at $37.0^{\circ} \mathrm{C}$ in the expanded BP I (arrow B), even after removing the field, BP I does not appear, and the cholesteric phase remains. This supports the fact that the expanded $\mathrm{BP}$ in the $\mathrm{LC} / \mathrm{PN}$ composite is not thermodynamically stable and is induced by the pinning effect on the $\mathrm{PN}$ surfaces in the $\mathrm{LC} / \mathrm{PN}$ composite.

Kikuchi et al. reported that a BP temperature is markedly extended by the polymerization of the defect portion of the BP LC [9]. Namely, they added a small amount of photopolymerizable monomers and a photoinitiator (less than $10 \mathrm{wt} \%$ ) to the BP LC and irradiated it with UV light in the temperature range of the BPs. As a result, the polymerized parts are separated from the LC and pushed out to the part containing disclinations. BP forms a 3D helical structure and inevitably has disclination lines. The existence of the disclinations should increase the elastic energy and destabilize the BP. Therefore, the replacement of disclination with a polymer should reduce the region with high elastic energy and stabilize the BP. On the other hand, our composite contains a large amount of monomers (32 wt\%) and forms a large-scale PN as compared with the BP lattice constant, as shown in Fig. 1. Therefore, the mechanism of the $\mathrm{BP}$ expansion in the $\mathrm{PN}$ is different from that of the polymer-stabilized system reported by Kikuchi.

\section{CONCLUSIONS}

The temperature range in which the $\mathrm{BP}$ appears was expanded at a cooling stage upon the introduction of a $\mathrm{PN}$. On the heating stage, the expansion was small. Once the cholesteric or nematic phase was induced by the application of an electric field in the extended temperature range, the $\mathrm{BP}$ was not restored even after removing the field. From these results, we can conclude that the expanded $\mathrm{BP}$ in the $\mathrm{LC} / \mathrm{PN}$ composite is not thermodynamically stable and is induced by the pinning effect on surfaces of the PN in the $\mathrm{LC} / \mathrm{PN}$ composite. 


\section{Acknowledgments}

We acknowledge Chisso Co. Ltd. for kindly providing the nematic liquid crystal material. We also thank
Dr. Yasuhiro Haseba of Chisso Co. Ltd. for helpful discussions. This study is supported by a Grant-in-Aid for Scientific Research from the Ministry of Education, Culture, Sports, Science and Technology of Japan.
[1] D. C. Wright and N. D. Mermin, Rev. Mod. Phys. 61, 385 (1989).

[2] P. P. Crooker, in Chirality in Liquid Crystals, Eds. H.-S. Kitzerow and Ch. Bahr (Springer, New York, 2001), p. 186-222.

[3] P. Etchegoin, Phys. Rev. E 62, 1435 (2000).

[4] W. Cao, A. Munoz, P. Palffy-Muhoray, and B. Taheri, Nat. Mater. 1, 111 (2002).

[5] S. Yokoyama, S. Mashiko, H. Kikuchi, K. Uchida, and T. Nagamura, Adv. Mater. 18, 48 (2006).

[6] Y. Hisakado, H. Kikuchi, T. Nagamura, and T. Kajiyama, Adv. Mater. 17, 2311 (2005).
[7] Y. Haseba, H. Kikuchi, T. Nagamura, and T. Kajiyama, Adv. Mater. 17, 2311 (2005)

[8] Y. Haseba and H. Kikuchi, Mol. Cryst. Liq. Cryst. 470, $1,(2007)$.

[9] H. Kikuchi, M. Yokota, Y. Hisakado, H. Yang, and T. Kajiyama, Nat. Mater. 1, 64 (2002).

[10] H. J. Coles and M. N. Pivnenko, Nature 436, 997 (2005).

[11] A. Yoshizawa, M. Sato, and J. Rokunohe, J. Mater. Chem. 15, 3285 (2005).

[12] H. Stegemeyer and F. Porsch, Phys. Rev. A 30, 3369 (1984). 